\title{
A NOTION OF FUNCTIONAL COMPLETENESS FOR FIRST-ORDER STRUCTURE
}

\author{
ETIENNE R. ALOMO TEMGOUA AND MARCEL TONGA
}

Received 27 September 2004 and in revised form 4 July 2005

Using $\star$-congruences and implications, Weaver (1993) introduced the concepts of prevariety and quasivariety of first-order structures as generalizations of the corresponding concepts for algebras. The notion of functional completeness on algebras has been defined and characterized by Burris and Sankappanavar (1981), Kaarli and Pixley (2001), Pixley (1996), and Quackenbush (1981). We study the notion of functional completeness with respect to $\star$-congruences. We extend some results on functionally complete algebras to first-order structures $\mathbf{A}=\left(A ; F^{\mathbf{A}} ; R^{\mathbf{A}}\right)$ and find conditions for these structures to have a compatible Pixley function which is interpolated by term functions on suitable subsets of the base set $A$.

\section{Introduction}

Functional completeness on algebras has been studied in $[2,3,4]$, and some results are given in [1]. Some basic notions in this field are listed in the definition below.

Definition 1.1. Let $\mathscr{A}=\left(A ; F^{\mathscr{A}}\right)$ be an algebra and let $f: A^{3} \rightarrow A$ be a function.

(i) $f$ is called a majority function if for all $a, b \in A, f(a, a, b)=f(b, a, a)=f(a, b, a)=$ a.

(ii) $f$ is called a Pixley function if for all $a, b \in A, f(a, b, b)=f(a, b, a)=f(b, b, a)=a$.

(iii) The ternary function $\mathbf{d}: A^{3} \rightarrow A$ defined by $\mathbf{d}(a, a, c)=c$ and $\mathbf{d}(a, b, c)=a$ if $a \neq b$ is called the discriminator function on $A$.

Moreover, if $\mathscr{A}$ is a finite nontrivial algebra, then $\mathscr{A}$ is called

(iv) primal if every $n$-ary function on $A, n \geq 1$, is a term function of $\mathscr{A}$;

(v) quasiprimal if the discriminator function on $A$ is a term function of $\mathscr{A}$;

(vi) functionally complete if every $n$-ary function on $A$ is a polynomial function of $\mathscr{A}$.

Our aim here is to formulate and characterize a notion of functionally complete firstorder structure $\mathbf{A}=\left(A ; F^{\mathbf{A}} ; R^{\mathbf{A}}\right)$, which takes care (in some sense) of the relations in $R^{\mathbf{A}}$.

Throughout, $\mathbf{A}=\left(A ; F^{\mathbf{A}} ; R^{\mathbf{A}}\right)$ is a nontrivial first-order structure. We denote by Con $(\mathbf{A})$ the set of congruences of $\mathbf{A}$. 
Definition 1.2 [7]. An element $\theta \in \operatorname{Con}(\mathbf{A})$ is called a $\star$-congruence if for any $m$-ary $r$ in $R$ and any pairs $\left\langle a_{i}, b_{i}\right\rangle \in \theta$ for $1 \leq i \leq m,\left\langle a_{1}, \ldots, a_{m}\right\rangle \in r^{\mathrm{A}}$ if and only if $\left\langle b_{1}, \ldots, b_{m}\right\rangle \in r^{\mathrm{A}}$.

$\mathrm{Con}_{\star}(\mathbf{A})$ will denote the set of $\star$-congruences of $\mathbf{A}$; it is easy to see that $\operatorname{Con}_{\star}(\mathbf{A})$ is a sublattice of $\operatorname{Con}(\mathbf{A})$; in fact $\operatorname{Con}_{\star}(\mathbf{A})$ is a complete lattice, and its largest element denoted by $1_{\mathrm{A}}$ is generally different from $A^{2}=\nabla_{A}$.

Let $\triangle_{A}$ be the smallest congruence of $\mathbf{A}$; when $\triangle_{A} \subsetneq 1_{\mathbf{A}} \subsetneq \nabla_{A}$, $\mathbf{A}$ is not simple, and the discriminator function $\mathbf{d}$ on $A$ is not a term function of $\mathbf{A}$. However, $\mathbf{d}$ may be interpolated by term functions on some parts of $A$.

For each $a$ in $A$, the $1_{\mathrm{A}}$ class of $a$ will be denoted by $\bar{a}$.

Definition 1.3 [6]. Let $\mathbf{A}$ be a structure, and let $f: A^{n} \rightarrow A, n \geq 1$, be an $n$-ary function.

(i) $f$ is said to be $1_{\mathrm{A}}$ compatible if $1_{\mathrm{A}}$ is a congruence of $(A ; f)$; that is, for any pairs $\left\langle a_{i}, b_{i}\right\rangle \in 1_{\mathrm{A}}$ for $1 \leq i \leq n,\left\langle f\left(a_{1}, \ldots, a_{n}\right), f\left(b_{1}, \ldots, b_{n}\right)\right\rangle \in 1_{\mathrm{A}}$.

(ii) $f$ is said to be termal on classes if for each $a \in A$, there is an $n$-ary term $t_{a}$ such that $f$ and $t_{a}^{\mathrm{A}}$ coincide on $\bar{a}$.

(iii) $f$ is said to be term representable on classes if there is an $n$-ary term $t$ such that $f$ and $t^{\mathrm{A}}$ coincide on every $1_{\mathrm{A}}$ class.

(iv) Let $\mathbf{A}$ be finite; then $\mathbf{A}$ is said to be $\star$-primal if every $1_{\mathbf{A}}$ compatible $n$-ary function on $A$ is term representable on classes.

(v) Let $\mathbf{A}$ be finite; then $\mathbf{A}$ is said to be $\star$-quasi-primal if the discriminator function on $A$ is term representable on classes.

We note that a unary function $f$ which is term representable on classes is a term function.

For any elements $a, b \in A$, let $\theta(a, b)$ be the principal congruence on $\mathbf{A}$ generated by $\langle a, b\rangle$; if $a, b \in A^{n}$, let $\operatorname{Cong}(a, b):=\bigvee_{1 \leq i \leq n} \theta(a(i), b(i))$.

For any $a^{1}, \ldots, a^{m} \in \bigcup_{a \in A} \bar{a}^{n}$, let $a_{i}:=\left\langle a^{1}(i), \ldots, a^{m}(i)\right\rangle$ for $1 \leq i \leq n$; then $B\left(a_{1}, \ldots, a_{n}\right)$ $:=\left\{x \in A^{m} ;\langle x(k), x(l)\rangle \in \operatorname{Cong}\left(a^{k}, a^{l}\right)\right.$ for $\left.1 \leq k, l \leq m\right\}$ is a subuniverse of $\mathbf{A}^{m}$.

The next theorem characterizes $\star$-primality.

Theorem 1.4 [6]. A finite structure $\mathbf{A}$ is $\star$-primal if and only if the following properties are satisfied.

(i) The only subuniverses of $\mathbf{A}^{2}$ are $\triangle_{A}, 1_{\mathbf{A}}$, and $\nabla_{A}$.

(ii) For any nonzero natural numbers $m, n$, and elements $a^{1}, \ldots, a^{m} \in \bigcup_{a \in A} \bar{a}^{n}$, if $a_{i}:=$ $\left\langle a^{1}(i), \ldots, a^{m}(i)\right\rangle$ for $1 \leq i \leq n$, then $B\left(a_{1}, \ldots, a_{n}\right)=\operatorname{Sg}\left(a_{1}, \ldots, a_{n}\right)$ as subuniverses of $\mathbf{A}^{m}$.

Condition (i) in this theorem implies that $\mathbf{A}$ is minimal (i.e., it has no proper substructure) and rigid (i.e., $\mathrm{id}_{A}$ is the only automorphism of $\mathbf{A}$ ).

\section{2. $\star$-Functionally complete structure}

Given a structure $\mathbf{A}=\left(A ; F^{\mathbf{A}} ; R^{\mathbf{A}}\right)$, we will make use of the structure (of different type) $\mathbf{A}_{A}:=\left(A ; F^{\mathbf{A}} \cup\left\{c_{a} ; a \in A\right\} ; R^{\mathbf{A}}\right)$ by adding a constant function with value $a$ for each element $a$ of $A$. So terms of $\mathbf{A}_{A}$ are exactly polynomials of $\mathbf{A}$. 
We can rephrase Definition 1.3(ii), (iii), and (iv) in terms of polynomials and obtain the following definition.

Definition 2.1. (i) $f$ is said to be polynomial on classes if for each $a \in A$, there is an $n$-ary polynomial $p_{a}$ such that $f$ and $p_{a}^{\mathrm{A}}$ coincide on $\bar{a}$.

(ii) $f$ is said to be polynomially representable on classes if there is an $n$-ary polynomial $p$ such that $f$ and $p^{\mathbf{A}}$ coincide on each class $\bar{a}$.

(iii) Let $\mathbf{A}$ be finite; $\mathbf{A}$ is said to be $\star$-functionally complete if any $n$-ary $1_{\mathbf{A}}$ compatible function on $A$ is polynomially representable on classes.

The following results give a relationship between $\star$-primality and $\star$-functional completeness; the first one is a direct consequence of the definitions.

Theorem 2.2. $\mathbf{A}$ is $\star$-functionally complete if and only if $\mathbf{A}_{A}$ is $\star$-primal.

Theorem 2.3. A is $\star$-primal if and only if each subuniverse of $\mathbf{A}^{m}, m \geq 2$, contains the set $\Delta_{A}(m):=\{(a, a, \ldots, a): a \in A\}$ and $\mathbf{A}$ is $\star$-functionally complete.

Proof. "If" part. Any constant function on $A$ is a term function of $\mathbf{A}$. Let $C$ be a subuniverse of $\mathbf{A}^{m}, m \geq 2$. Then there is some $u=\left\langle u_{1}, u_{2}, \ldots, u_{m}\right\rangle$ in $\mathbf{A}^{m}$ such that $u \in C$. Let $a \in A$, the constant function with value $a$ is representable by a term $t$ on $A$. Then $\langle a, a, \ldots, a\rangle=\left\langle t\left(u_{1}\right), \ldots, t\left(u_{m}\right)\right\rangle=t^{\mathbf{A}^{m}}\left(\left\langle u_{1}, \ldots, u_{m}\right\rangle\right) \in C$. So $\Delta_{A}(m) \subseteq C$.

"only if" part. Let $f$ be a $1_{A}$ compatible $n$-ary function. Then $f$ is representable by a polynomial $p\left(x_{1}, \ldots, x_{n}, c_{a_{1}}, \ldots, c_{a_{m}}\right)$ on classes. Let $A=\left\{b_{1}, \ldots, b_{k}\right\}$; then $\left\langle a_{i}, \ldots, a_{i}\right\rangle \in$ $\operatorname{Sg}\left(\left\langle b_{1}, \ldots, b_{k}\right\rangle\right) \subseteq A^{k}$. There is a unary term $t_{i}$ such that $t_{i}\left(b_{j}\right)=a_{i}$ for $1 \leq j \leq k$. So $t_{i}$ represents $c_{a_{i}}$ on $A$.

The term $q\left(x_{1}, \ldots, x_{n}\right)=p\left(x_{1}, \ldots, x_{n}, t_{1}\left(x_{1}\right), \ldots, t_{m}\left(x_{1}\right)\right)$ represents $f$ on classes.

Now we introduce some important ideas on $\star$-functional completeness.

Lemma 2.4. Let $\mathbf{A}$ be a $\star$-functionally complete structure and let $C$ be a subuniverse of $1_{\mathbf{A}}$ which is a subdirect product of $\mathbf{A}^{2}$ such that the projection $\pi_{i}: C \rightarrow A$ is not an isomorphism for $i=1$ or $i=2$. Then there is some $b$ in $A$ such that $\langle y, b\rangle \in C$ for all $y \in \bar{b}$.

Proof. Suppose that $\pi_{2}: C \rightarrow A$ is not one-to-one. Then there are $\left\langle a_{1}, b\right\rangle,\left\langle a_{2}, b\right\rangle \in C$ such that $a_{1} \neq a_{2}$. Let $n$ be a natural number such that $2^{n} \geq|\bar{b}|$. Let $f: A^{n} \rightarrow A$ be a function which satisfies the following conditions.

(i) $f\left(x_{1}, \ldots, x_{n}\right)=x_{1}$ if $\left\langle x_{i}, x_{j}\right\rangle \notin 1_{\mathrm{A}}$ for some $i<j$.

(ii) $f\left(\bar{a}^{n}\right) \subseteq \bar{a}$ for each $a \in A$.

(iii) $f\left(\left\{a_{1}, a_{2}\right\}^{n}\right)=\bar{b}$.

$f$ is $1_{\mathrm{A}}$ compatible. Let $t\left(x_{1}, \ldots, x_{n}, c_{u_{1}}, \ldots, c_{u_{m}}\right)$ be a polynomial representing $f$ on classes. For $1 \leq i \leq m$, let $b_{i} \in A$ such that $\left\langle u_{i}, b_{i}\right\rangle \in C$.

Let $b^{\prime}:=t\left(b, \ldots, b, b_{1}, \ldots, b_{m}\right)$; if $y \in \bar{b}$, then there are $d_{1}, \ldots, d_{n} \in\left\{a_{1}, a_{2}\right\}$ such that $y=f\left(d_{1}, \ldots, d_{n}\right)$. So $y=t\left(d_{1}, \ldots, d_{n}, u_{1}, \ldots, u_{m}\right)$; and

$$
\begin{aligned}
\left\langle y, b^{\prime}\right\rangle & =\left\langle t\left(d_{1}, \ldots, d_{n}, u_{1}, \ldots, u_{m}\right), t\left(b, \ldots, b, b_{1}, \ldots, b_{m}\right)\right\rangle \\
& =t^{\mathbf{A}^{(n+m)}}\left(\left\langle d_{1}, b\right\rangle, \ldots,\left\langle d_{n}, b\right\rangle,\left\langle u_{1}, b_{1}\right\rangle, \ldots,\left\langle u_{m}, b_{m}\right\rangle\right) \in C .
\end{aligned}
$$

So, $\overline{b^{\prime}}=\bar{b}$ and $\left\langle y, b^{\prime}\right\rangle \in C$ for all $y \in \bar{b}$. 
2210 A notion of functional completeness for first-order structure

Lemma 2.5. If $\mathbf{A}$ is $\star$-functionally complete and minimal, then every subuniverse of $1_{\mathbf{A}}$ either is the graph of an automorphism of $\mathbf{A}$ or contains $\triangle_{A}$ properly.

Proof. Let $C$ be a subuniverse of $1_{\mathbf{A}}$. Since $\mathbf{A}$ is minimal, $C$ is a subdirect product of $\mathbf{A}^{2}$.

Suppose that $C$ is the graph of a permutation $\alpha$ of $A$.

Let $f$ be an $n$-ary operation of $\mathbf{A}$; then $\left\langle a_{1}, \alpha\left(a_{1}\right)\right\rangle, \ldots,\left\langle a_{n}, \alpha\left(a_{n}\right)\right\rangle \in C$ implies that $\left\langle f\left(a_{1}, \ldots, a_{n}\right), f\left(\alpha\left(a_{1}\right), \ldots, \alpha\left(a_{n}\right)\right)\right\rangle \in C$, thus $f\left(\alpha\left(a_{1}\right), \ldots, \alpha\left(a_{n}\right)\right)=\alpha\left(f\left(a_{1}, \ldots, a_{n}\right)\right)$. Also, let $r$ be an $m$-ary relation of $\mathbf{A}$; since $C$ is a subuniverse of $1_{\mathrm{A}}$, for any elements $\left\langle a_{1}\right.$, $\left.\alpha\left(a_{1}\right)\right\rangle, \ldots,\left\langle a_{m}, \alpha\left(a_{m}\right)\right\rangle \in C,\left(a_{1}, \ldots, a_{m}\right) \in r$ if and only if $\left(\alpha\left(a_{1}\right), \ldots, \alpha\left(a_{m}\right)\right) \in r$. Therefore $\alpha$ is an automorphism of $\mathbf{A}$.

If $C$ is not the graph of a permutation of $A$, it contains two elements $\left\langle a_{1}, b\right\rangle$ and $\left\langle a_{2}, b\right\rangle$ with $a_{1} \neq a_{2}$, or two elements $\left\langle a, b_{1}\right\rangle$ and $\left\langle a, b_{2}\right\rangle$ with $b_{1} \neq b_{2}$. Then from Lemma 2.4, there is some $b$ such that $\bar{b} \times\{b\} \subseteq C$; so $\langle b, b\rangle \in C$. Since $\mathbf{A}$ has no proper subuniverse, $\triangle_{A}$ is contained in $C$.

THeorem 2.6. If $\mathbf{A}$ is $\star$-functionally complete and minimal, then there is a unary term $t$ such that for each $\langle a, b\rangle \in t\left(1_{\mathbf{A}}\right)$, there is an automorphism $\sigma$ of $\mathbf{A}$ such that $\sigma(a)=b$.

Proof. Let $B=\{|t(A)| ; t$ an unary term $\}$; then $B \subseteq N^{*}$. Let $n_{0}=\min (B)$ and let $t_{0}$ be a unary term with $\left|t_{0}(A)\right|=n_{0}$. Let $\langle a, b\rangle \in t_{0}\left(1_{\mathrm{A}}\right)$; then $C=\operatorname{Sg}(\langle a, b\rangle)$ is a subuniverse of $1_{\mathrm{A}}$. From Lemma 2.5, $C$ is the graph of an automorphism of $\mathbf{A}$ or $\triangle_{A}$ is a proper subset of $C$.

If $\triangle_{A} \subsetneq C$, then there is a unary term $t_{1}$ such that $t_{1}(a)=t_{1}(b)$; thus $\left|t_{1} t_{0}(A)\right|<n_{0}$, a contradiction. Therefore $C$ is the graph of an automorphism $\sigma$ of $\mathbf{A}$, and $\sigma(a)=b$.

Corollary 2.7. If $\mathbf{A}$ is $\star$-functionally complete and minimal, and there is a unary term $t$ and $a \in A$ such that $t(A) \subseteq \bar{a}$; then there is a unary term $t_{0}$ such that for all $b, c \in t_{0}(A)$, there is an automorphism $\sigma$ of $\mathbf{A}$ such that $\sigma(b)=c$.

Proof. Let $B:=\{|t(A)| ; t$ a unary term and $t(A) \subseteq \bar{a}\}$; then the result follows by using the same argument as in the proof of Theorem 2.6.

\section{Interpolation of Pixley functions}

In this section, we examine some links between $\star$-functional completeness and term interpolation of $1_{\mathrm{A}}$ compatible Pixley functions.

Theorem 3.1. If $\mathbf{A}$ is $\star$-functionally complete and minimal, then for each $a \in A$, there is a $1_{\mathrm{A}}$ compatible Pixley function which is representable by a term on $\bar{a}$.

Proof. Let $d \in A$ be fixed, and let $p$ be the ternary function defined by

$$
p(a, b, c)= \begin{cases}a & \text { if }(\bar{a}=\bar{b}=\bar{c} \text { and } c \in\{a, b\}) \text { or }(\bar{a} \neq \bar{b}=\bar{c}) \\ c & \text { if }(\bar{a}=\bar{b}=\bar{c} \text { and } a=b) \text { or }(\bar{a} \in\{\bar{b}, \bar{c}\} \text { and } \bar{b} \neq \bar{c}) \\ b & \text { elsewhere. }\end{cases}
$$

$p$ is a $1_{\mathrm{A}}$ compatible Pixley function. So $p$ is representable by a polynomial $p_{0}(x, y, z):=$ $t\left(x, y, z, c_{a_{1}}, \ldots, c_{a_{m}}\right)$ on classes. Let $t_{0}$ be the unary term of Theorem 2.6. The subuniverse 
of A generated by $e=t_{0}(d)$ is $A$. There are $m$ unary terms $t_{1}, \ldots, t_{m}$ such that $a_{1}=$ $t_{1}(e), \ldots, a_{m}=t_{m}(e)$. So $p_{0}(x, y, z)=t\left(x, y, z, t_{1}(e), \ldots, t_{m}(e)\right)$.

The term $s(x, y, z, w):=t\left(x, y, z, t_{1}(w), \ldots, t_{m}(w)\right)$ is a 4 -ary term and $p(x, y, z)=$ $s(x, y, z, e)$ on classes. Consider the term $m(x, y, z):=s\left(x, y, z, t_{0}(x)\right)$ and $b, c \in \bar{d}$; then $\left\langle t_{0}(b), e\right\rangle \in t_{0}\left(1_{A}\right)$; from Theorem 2.6 there is an automorphism $\sigma$ of $\mathbf{A}$ such that $t_{0}(b)=$ $\sigma(e)$. Then $|\sigma(\bar{b})|=|\overline{\sigma(b)}|$; so,

$$
m(b, b, c)=s\left(b, b, c, t_{0}(b)\right)=s(b, b, c, \sigma(e))=\sigma s\left(\sigma^{-1} b, \sigma^{-1} b, \sigma^{-1} c, e\right)
$$

that is

$$
m(b, b, c)=\sigma p\left(\sigma^{-1} b, \sigma^{-1} b, \sigma^{-1} c\right)=\sigma \sigma^{-1}(c)=c
$$

\footnotetext{
$\bar{d}$.

Similarly, $m(b, c, b)=b$ and $m(b, c, c)=b$. So $m$ is a ternary term which is Pixley's on
}

Corollary 3.2. If $\mathbf{A}$ is $\star$-functionally complete and minimal, and there is a unary term $t(x)$ and $a \in A$ such that $t(A) \subseteq \bar{a}$, then $\mathbf{A}$ has a $1_{\mathrm{A}}$ compatible Pixley function which is term representable on classes.

The proof is similar to the proof of Theorem 3.1, using the unary term $t_{0}$ of Corollary 2.7.

A first-order structure $\mathbf{A}$ is $\star$-arithmetical if $\operatorname{Con}_{\star}(\mathbf{A})$ is arithmetical.

Let $K$ be a class of first-order structures, and consider the following classes.

(i) $H^{*}(K)$ is the class of $\star$-quotients of structures in $K$.

(ii) $S(K)$ is the class of substructures of structures in $K$.

(iii) $P(K)$ is the class of products of structures in $K$.

(iv) A $\star$-variety is a class of structures preserved by $H^{*}, S$, and $P$. So $H^{*} S P(K)$ is the $\star$-variety generated by $K$.

It is proved in [7] that a $\star$-variety $\mathscr{V}$ is $\star$-arithmetical if and only if there is a ternary term $q(x, y, z)$ such that for any $\mathbf{A}$ in $\mathscr{V}$ and $a, b \in A,\langle a, b\rangle \in 1_{\mathbf{A}}$ implies that $q^{\mathbf{A}}(a, b, a)=$ $q^{\mathbf{A}}(b, b, a)=q^{\mathbf{A}}(a, b, b)=a$.

This result says that there is a term which is Pixley's on $1_{\mathrm{A}}$ classes for each $\mathbf{A}$ in $\mathscr{V}$. The remark below gives some criteria for a $\star$-variety generated by a $\star$-functionally complete structure to be $\star$-arithmetical.

Remark 3.3. Suppose that $\mathbf{A}$ is $\star$-functionally complete, minimal, and there is a unary term $t$ and $a \in A$ such that $t(A) \subseteq \bar{a}$.

If for each $\mathbf{B} \in H^{*} S P(\mathbf{A})$, the ternary term $m(x, y, z)$ of Corollary 3.2 is a Pixley function on $1_{\mathbf{B}}$ classes, then $H^{*} S P(\mathbf{A})$ is $\star$-arithmetical; in particular, if $1_{\mathbf{B}} \subseteq\left(1_{\mathbf{A}}\right)^{I}$ for each non empty set $I$ and each $\mathbf{B} \subseteq \mathbf{A}^{I}$.

Theorem 3.4. If $\mathbf{A}$ is $\star$-functionally complete and minimal, and there is a unary term $t$ and $a \in A$ such that $t(A) \subseteq \bar{a}$, then $\mathbf{A}$ is $\star$-quasiprimal. 
2212 A notion of functional completeness for first-order structure

Proof. Let $q: A^{3} \rightarrow A$ be the function defined by

$$
q(a, b, c)= \begin{cases}a & \text { if } \bar{a}=\bar{b}=\bar{c}, a \neq b \\ c & \text { if not. }\end{cases}
$$

$q$ is $1_{\mathrm{A}}$ compatible. So $q$ is representable on classes by a polynomial $t\left(x, y, z, c_{b_{1}}, \ldots, c_{b_{k}}\right)$. Let $t_{0}$ be the unary term of Corollary 2.7 and $e \in t_{0}(A)$; then $\operatorname{Sg}(e)=A$. There are $k$ unary terms $t_{1}, \ldots, t_{k}$ such that $b_{1}=t_{1}(e), \ldots, b_{k}=t_{k}(e)$. So $q(x, y, z)=t\left(x, y, z, t_{1}(e), \ldots, t_{k}(e)\right)$ on classes. Consider the term $m^{\prime}(x, y, z):=t\left(x, y, z, t_{1}\left(t_{0}(x)\right), \ldots, t_{k}\left(t_{0}(x)\right)\right)$ and $a, b, c \in A$ such that $\bar{a}=\bar{b}=\bar{c}$. Then there is an automorphism $\sigma$ of A such that $t_{0}(a)=\sigma(e)$.

If $a \neq b$, then $m^{\prime}(a, b, c)=t\left(a, b, c, t_{1}\left(t_{0}(a)\right), \ldots, t_{k}\left(t_{0}(a)\right)\right)$, that is, $m^{\prime}(a, b, c)=$ $\sigma q\left(\sigma^{-1} a, \sigma^{-1} b, \sigma^{-1} c\right)=\sigma \sigma^{-1}(a)=a$.

Similarly, if $a=b$, then $m^{\prime}(a, b, c)=\sigma q\left(\sigma^{-1} a, \sigma^{-1} b, \sigma^{-1} c\right)=\sigma \sigma^{-1}(c)=c$.

So $m^{\prime}(x, y, z)$ represents the discriminator function on classes; and $\mathbf{A}$ is $\star$-quasiprimal.

THeORem 3.5. If $\mathbf{A}$ is $\star$-functionally complete, minimal, and there is a unary term $t$ such that $|t(A)|=1$, then $\mathbf{A}$ is $\star$-primal.

Proof. Using Theorem 2.3, we will show that any subuniverse of $\mathbf{A}^{m}$ contains $\triangle_{A}(m)$. Let $t(A)=\{a\}$; we have $\operatorname{Sg}(a)=A$. Let $C$ be a subuniverse of $\mathbf{A}^{m}$ and $u \in C ;\langle a, \ldots, a\rangle=$ $t^{\mathbf{A}^{m}}(u) \in C$. So $\triangle_{A}(m)=\operatorname{Sg}(\langle a, \ldots, a\rangle) \subseteq C$ because $\mathbf{A}$ is minimal.

\section{Some examples of $\star$-functionally complete structure}

We begin with a version of a Baker-Pixley lemma (see [1, Section IV-10]) suitable for our purpose.

LEMMA 4.1. Let $\mathbf{A}$ be a finite first-order structure such that there is a majority function which is term representable on classes and let $f: A^{n} \rightarrow A$ be an $n$-ary function, $n \geq 1$.

If for each nonzero natural number $m \leq\left|A / 1_{\mathbf{A}}\right|+1$, and any elements $a_{1}, \ldots, a_{m}$ in $\bigcup_{a \in A} \bar{a}^{n}, B=\operatorname{Sg}\left(\left\langle a_{1}(1), \ldots, a_{m}(1)\right\rangle, \ldots,\left\langle a_{1}(n), \ldots, a_{m}(n)\right\rangle\right)$ is preserved by $f$, then there is a term $p\left(x_{1}, \ldots, x_{n}\right)$ representing $f$ on classes.

The proof is similar to the proof of the original lemma in [1].

Example 4.2. Let $\mathbf{Z}=(\mathbb{Z} ;+,-, 0 ; \leq)$ be the ordered group of integers. Let $\theta$ be an equivalence relation on $\mathbb{Z} ; \theta$ is a congruence if and only if there is a natural number $n$ such that $\langle a, b\rangle \in \theta$ if and only if $a-b \in n \mathbb{Z}$.

Claim (i). We have $1_{\mathbf{Z}}=\triangle_{\mathbb{Z}}$.

Proof. Let $\theta$ be an equivalence relation on $\mathbb{Z}$ such that $\theta \neq \triangle_{\mathbb{Z}}$. Then there is some $\langle a, b\rangle \in$ $\theta$ such that $a \neq b$. Thus $a<b$ or $b<a$. By symmetry, we can consider only the first case; since $\langle a, b\rangle,\langle b, a\rangle \in \theta, a \leq b$ and $b \not \leq a, \theta$ is not $a \star$-congruence. So $\triangle_{\mathbb{Z}}$ is the only $\star$ congruence on $\mathbf{Z}$.

Let $A:=\mathbb{Z} / 2 \mathbb{Z}=\{0,1\}$ and $\mathbf{A}=(A ;+,-, 0 ; \rho)$, where $\rho$ is the binary relation defined by $\rho:=\{\langle 0,0\rangle,\langle 0,1\rangle,\langle 1,1\rangle\}$; then it is easy to see that $1_{\mathrm{A}}=\triangle_{A}$. 
Claim (ii). A is $\star$-functionally complete.

Proof. Let $h$ be an $n$-ary function on $\{0,1\}, n \geq 1$.

If $h(0, \ldots, 0)=0$ and $h(1, \ldots, 1)=0$, then $h$ is representable on classes by the polynomial $c_{0}$.

If $h(0, \ldots, 0)=0$ and $h(1, \ldots, 1)=1$, then $h$ is representable on classes by the first projection.

If $h(0, \ldots, 0)=1$ and $h(1, \ldots, 1)=0$, then $h$ is representable on classes by the polynomial $t\left(x_{1}, \ldots, x_{n}\right):=x_{1}+c_{1}$.

If $h(0, \ldots, 0)=1$ and $h(1, \ldots, 1)=1$, then $h$ is representable on classes by the polynomial $c_{1}$.

Therefore $h$ is polynomially representable on classes.

Since $\{0\}$ is a subuniverse the $\mathbf{A}, \mathbf{A}$ is not $\star$-primal.

The function $m$ defined on $A$ by

$$
m(a, b, c)= \begin{cases}a & \text { if } a=b \text { or } a=c \\ b & \text { if } b=c \\ c & \text { elsewhere }\end{cases}
$$

is not a polynomial function of $\mathscr{A}=(A ;+,-, 0)$. So $\mathscr{A}$ is not functionally complete.

Example 4.3. Consider the set $A=\{a, b, c\}$ and the operations $f, g$ on $A$ defined as follows:

$$
f(x, y, z, u)= \begin{cases}\mathbf{d}(x, y, z) & \text { if } u=a \text { and } \\ & ((x, y, z \in\{b, c\}) \text { or }(x=a \neq y) \text { or }(y=a \neq x)) \\ a & \text { if not. }\end{cases}
$$

$g(a)=a, g(b)=c$, and $g(c)=b$.

Consider the structure $\mathbf{A}=(A ; f, g, a ; r)$, where $r=\{\langle a, b\rangle,\langle a, c\rangle,\langle b, a\rangle,\langle c, a\rangle\}$. It is easy to see that $\operatorname{Con}(\mathbf{A})=\left\{\triangle_{A}, \theta, \nabla_{A}\right\}$, where $\theta=\triangle_{A} \cup\{\langle b, c\rangle,\langle c, b\rangle\}$. Since $\langle a, a\rangle,\langle b, a\rangle \in$ $\nabla_{A},\langle a, b\rangle \in r$ and $\langle a, a\rangle \notin r, \nabla_{A}$ is not a $\star$-congruence. We can easily verify that $\theta$ is a $\star$-congruence; thus $1_{\mathbf{A}}=\theta$, and $|A / \theta|=2$.

We prove that $\mathbf{A}_{A}$ is $\star$-primal.

We have $\operatorname{Con}\left(\mathbf{A}_{A}\right)=\operatorname{Con}(\mathbf{A})$; so $1_{\mathbf{A}_{A}}=\theta$. The term $f\left(x, y, z, c_{a}\right)$ represents the discriminator function on classes. Let $h$ be an $n$-ary function on $A, n \geq 1$, which preserves $\theta$; using Lemma 4.1, we will prove that for each nonzero natural number $m \leq 3$, for all elements $a_{1}, \ldots, a_{m}$ in $\bigcup_{a \in A} \bar{a}^{n}, h$ preserves the subuniverse $B=\operatorname{Sg}\left(\left\langle a_{1}(1), \ldots, a_{m}(1)\right\rangle, \ldots,\left\langle a_{1}(n), \ldots\right.\right.$, $\left.\left.a_{m}(n)\right\rangle\right)$ of $\left(\mathbf{A}_{A}\right)^{m}$.

(i) If $m=1$, then $B=A$ ( $\mathbf{A}_{A}$ is minimal); so $h$ preserves $B$.

(ii) If $m=2$, then the following hold. 


\section{A notion of functional completeness for first-order structure}

If $B$ is not a subset of $\theta$, then there is $\langle x, y\rangle \in B$ such that $\langle x, y\rangle \notin \theta$; since $g$ preserves $B$, we have $\{\langle a, b\rangle,\langle a, c\rangle\} \subseteq B$ or $\{\langle b, a\rangle,\langle c, a\rangle\} \subseteq B$. By symmetry, we can consider only the first case.

Since $\langle b, c\rangle=f(\langle b, b\rangle,\langle a, b\rangle,\langle a, c\rangle,\langle a, a\rangle) \in B,\langle c, b\rangle=g(\langle b, c\rangle) \in B$, and $\langle b, a\rangle=$ $f(\langle b, b\rangle,\langle a, b\rangle,\langle a, a\rangle,\langle a, a\rangle) \in B$, so $\langle c, a\rangle=g(\langle b, a\rangle) \in B$; therefore $B=A^{2}$.

If $\triangle_{A} \varsubsetneqq B \subseteq \theta$, then there is $\langle x, y\rangle \in B$ such that $x \neq y$; so $\langle x, y\rangle=\langle b, c\rangle$ or $\langle x, y\rangle=$ $\langle c, b\rangle$. Since $\langle b, c\rangle \in B$ if and only if $\langle c, b\rangle \in B$, we have $B=\theta$. Therefore, $B$ is one of the subuniverses $\triangle_{A}, \theta$, and $\nabla_{A}$. Thus $h$ preserves $B$.

(iii) If $m=3$, for $1 \leq i \leq 3$, we denote by $\left\|a_{i}\right\|$ the set $\left\{a_{i}(1), \ldots, a_{i}(n)\right\}$.

If $\left\|a_{1}\right\|=\{a\},\left\|a_{2}\right\|=\{a\}$, and $\left\|a_{3}\right\|=\{a\}, B=\triangle_{A}(3)$ and $h$ preserves $B$.

If $\left\|a_{1}\right\|=\{a\},\left\|a_{2}\right\|=\{a\}$, and $\left\|a_{3}\right\|=\{b\}$, then $\langle a, a, b\rangle \in B$; so $\langle a, a, c\rangle=g(\langle a, a, b\rangle) \in$ $B$, and $\langle b, b, a\rangle=f(\langle b, b, b\rangle,\langle a, a, b\rangle,\langle a, a, a\rangle,\langle a, a, a\rangle) \in B$; moreover, $\langle c, c, a\rangle=$ $g(\langle b, b, a\rangle),\langle b, b, c\rangle=f(\langle b, b, b\rangle,\langle a, a, b\rangle,\langle a, a, c\rangle,\langle a, a, a\rangle)$, and $\langle c, c, b\rangle=g(\langle b, b, c\rangle)$ are in $B$; thus $B=\triangle_{A} \times A$ and $h$ preserves $B$.

If $\left\|a_{1}\right\|=\{a\},\left\|a_{2}\right\|=\{a\}$, and $\left\|a_{3}\right\|=\{c\}$, then $B=\triangle_{A} \times A$.

If $\left\|a_{1}\right\|=\{a\},\left\|a_{2}\right\|=\{a\}$, and $\left\|a_{3}\right\|=\{b, c\}$, then $B=\triangle_{A} \times A$.

If $\left\|a_{1}\right\|=\{a\},\left\|a_{2}\right\|=\{b\}$, and $\left\|a_{3}\right\|=\{c\}$, then $\langle a, b, c\rangle \in B$, and $\langle a, c, b\rangle=g(\langle a, b, c\rangle) \in$ $B,\langle b, a, a\rangle=f(\langle b, b, b\rangle,\langle a, b, c\rangle,\langle a, a, a\rangle,\langle a, a, a\rangle) \in B$, and $\langle c, a, a\rangle=g(\langle b, a, a\rangle) \in B$; $\langle a, b, b\rangle=f(\langle b, b, b\rangle,\langle b, a, a\rangle,\langle a, a, a\rangle,\langle a, a, a\rangle) \in B$, and $\langle a, c, c\rangle \in B ;\langle b, c, c\rangle=f(\langle b, b, b\rangle$, $\langle a, b, b\rangle,\langle a, c, c\rangle,\langle a, a, a\rangle) \in B$, and $\langle c, b, b\rangle \in B ;\langle b, c, b\rangle=f(\langle b, b, b\rangle,\langle a, b, c\rangle,\langle b, c, c\rangle$, $\langle a, a, a\rangle) \in B$, and $\langle c, b, c\rangle \in B ;\langle b, b, c\rangle=f(\langle b, b, b\rangle,\langle a, b, b\rangle,\langle c, b, c\rangle,\langle a, a, a\rangle) \in B$, and $\langle c, c, b\rangle \in B$.

Thus $B=\left\{(x, y, z) \in A^{3} ;\langle y, z\rangle \in \theta\right\}$. So $h$ preserves $B$.

Let $\varepsilon$ be a permutation of $\{1,2,3\}$; the function $\alpha_{\varepsilon}: A^{3} \rightarrow A^{3}$ defined by $\alpha_{\varepsilon}\left(x_{1}, x_{2}, x_{3}\right)=$ $\left(x_{\varepsilon(1)}, x_{\varepsilon(2)}, x_{\varepsilon(3)}\right)$ is an automorphism of $\mathbf{A}^{3}$.

Therefore the subuniverse $B$ is one of the elements of the set $E$, where $E=\bigcup\left\{\alpha_{\varepsilon}\left(\left\{\triangle_{A}(3)\right.\right.\right.$, $\left.\left.\triangle_{A} \times A, A \times \theta\right\}\right) ; \varepsilon$ a permutation of $\left.\{1,2,3\}\right\}$. Thus $h$ preserves $B$. So $h$ is term representable on classes and $\mathbf{A}_{A}$ is $\star$-primal.

The set $B:=\{\langle a, a\rangle,\langle a, b\rangle,\langle a, c\rangle\}$ is a subuniverse of $\mathbf{A}^{2}$; thus $\mathbf{A}$ is not $\star$-primal.

We prove that $D:=\{\langle a, a, b\rangle,\langle b, c, a\rangle,\langle a, a, c\rangle,\langle c, b, a\rangle,\langle a, a, a\rangle\}$ is a subuniverse of $\mathbf{A}^{3}$.

If $x \in\{\langle a, a, b\rangle,\langle a, a, c\rangle,\langle a, a, a\rangle\}$ and $y, u, v$ are in $D$, then $f(x, y, u, v) \in\{\langle a, a, b\rangle$, $\langle a, a, c\rangle,\langle a, a, a\rangle\} \subseteq D$.

If $x, v$ are in $\{\langle b, c, a\rangle,\langle c, b, a\rangle\}$ and $y, u$ are in $D$, then $f(x, y, u, v) \in\{\langle a, a, b\rangle,\langle a, a, c\rangle$, $\langle a, a, a\rangle\} \subseteq D$.

If $x=\langle b, c, a\rangle, y \in\{\langle a, a, b\rangle,\langle a, a, c\rangle\}, u \in D$, and $v$ is an element of $\{\langle a, a, b\rangle,\langle a, a, c\rangle$, $\langle a, a, a\rangle\}$, then $f(x, y, u, v)=\langle b, c, a\rangle \in D$.

If $x=\langle b, c, a\rangle, \quad y=\langle b, c, a\rangle, u \in\{\langle b, c, a\rangle,\langle c, b, a\rangle\}$, and $v \in\{\langle a, a, b\rangle,\langle a, a, c\rangle$, $\langle a, a, a\rangle\}$, then $f(x, y, u, v) \in\{\langle b, c, a\rangle,\langle c, b, a\rangle\} \subseteq D$.

If $x=\langle b, c, a\rangle, y=\langle c, b, a\rangle$, and $u, v \in\{\langle a, a, b\rangle,\langle a, a, c\rangle,\langle a, a, a\rangle t\}$, then $f(x, y, u, v) \in$ $\{\langle a, a, b\rangle,\langle a, a, c\rangle,\langle a, a, a\rangle\} \subseteq D$.

If $x=\langle b, c, a\rangle, y=\langle c, b, a\rangle, u \in\{\langle b, c, a\rangle,\langle c, b, a\rangle\}$, and $v \in\{\langle a, a, b\rangle,\langle a, a, c\rangle,\langle a, a, a\rangle\}$, then $f(x, y, u, v)=\langle b, c, a\rangle \in D$.

We can also show that if $x=\langle c, b, a\rangle$ and $y, u, v \in D$, then $f(x, y, u, v) \in D$. Therefore $D$ is a subuniverse of $\mathbf{A}^{3}$. 
The function $h: A^{2} \rightarrow A$ defined by

$$
h(x, y)= \begin{cases}y & \text { if } \bar{x}=\bar{y} \\ b & \text { if }(x=a \text { and } y=b) \text { or }(x=c \text { and } y=a) \\ c & \text { elsewhere }\end{cases}
$$

is $\theta$ compatible. The subuniverses of $\mathbf{A}^{2}$ are $\triangle_{A}, C=\{\langle b, c\rangle,\langle a, a\rangle,\langle c, b\rangle\}, \theta,\{a\} \times A, A \times$ $\{a\}$ and $A^{2}$; so $h$ preserves the subuniverses of $\mathbf{A}^{2}$. Since $\langle b, c, c\rangle=h(\langle a, a, b\rangle,\langle b, c, a\rangle) \notin$ $D$, using [1, Lemma IV-10.4], we see that there is no majority term for $\mathbf{A}$.

The algebra $\mathbf{A}=(A ; f, g, a)$ is not primal since $\{a\}$ is a subuniverse of $\mathbf{A}$. Con $(\mathbf{A})=$ $\left\{\triangle_{A}, \theta, \nabla_{A}\right\}$, thus $\mathbf{A}$ is not quasiprimal and is not functionally complete.

\section{Acknowledgment}

We would like to thank the referees for pointing out several mistakes in the first version of the work.

\section{References}

[1] S. Burris and H. P. Sankappanavar, A Course in Universal Algebra, Graduate Texts in Mathematics, vol. 78, Springer, New York, 1981.

[2] K. Kaarli and A. F. Pixley, Polynomial Completeness in Algebraic Systems, Chapman \& Hall/CRC, Florida, 2001.

[3] A. F. Pixley, Boolean universal algebra, Logic and Algebra (Pontignano, 1994) (A. Ursini and P. Agliano, eds.), Lecture Notes in Pure and Appl. Math., vol. 180, Marcel Dekker, New York, 1996, pp. 245-266.

[4] R. W. Quackenbush, A new proof of Rosenberg's primal algebra characterization theorem, Finite Algebra and Multiple-Valued Logic (Szeged, 1979), Colloq. Math. Soc. János Bolyai, vol. 28, North-Holland, Amsterdam, 1981, pp. 603-634.

[5] M. Tonga, Strong congruences and Mal'cev conditions on first order structures, Contributions to General Algebra, 13 (Velké Karlovice, 1999/Dresden, 2000), Johannes Heyn, Klagenfurt, 2001, pp. 335-344.

[6] M. Tonga and E. R. Temgoua Alomo, A notion of primality for first order structures, 65 th Workshop on General Algebra, 18th Conference for Young Algebraists, March 2003, University of Potsdam, Potsdam, 2003.

[7] N. Weaver, Generalized varieties, Algebra Universalis 30 (1993), no. 1, 27-52.

Etienne R. Alomo Temgoua: Department of Mathematics, École Normale Supérieure, University of Yaoundé-1, P.O. Box 47, Yaoundé, Cameroon

E-mail address: rtemgoua@uycdc.uninet.cm

Marcel Tonga: Department of Mathematics, Faculty of Science, University of Yaoundé-1, P.O. Box 812 , Yaoundé, Cameroon

E-mail address: mtonga@uycdc.uninet.cm 


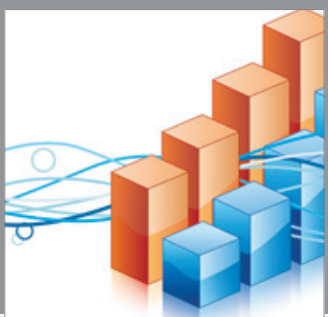

Advances in

Operations Research

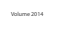

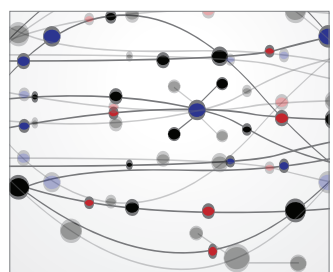

\section{The Scientific} World Journal
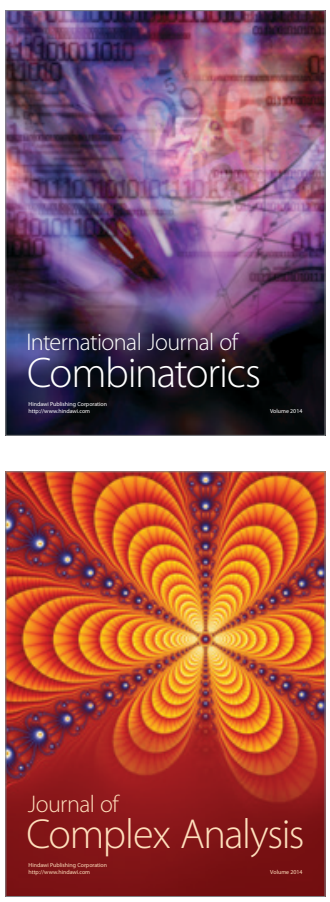

International Journal of

Mathematics and

Mathematical

Sciences
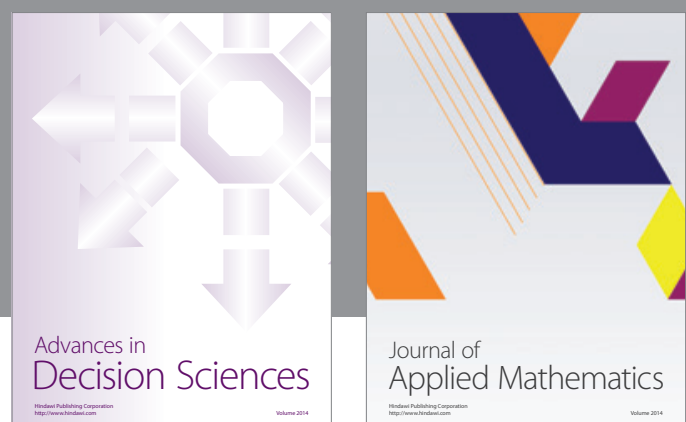

Journal of

Applied Mathematics
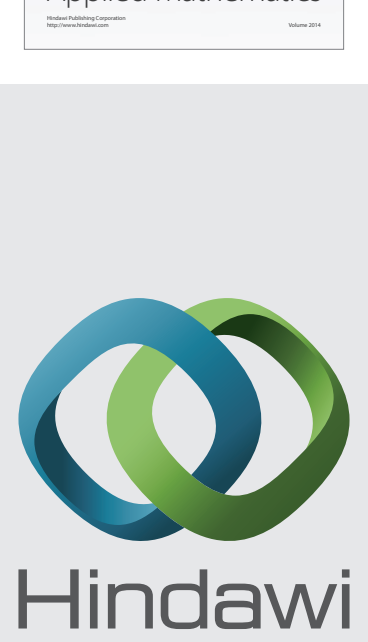

Submit your manuscripts at http://www.hindawi.com
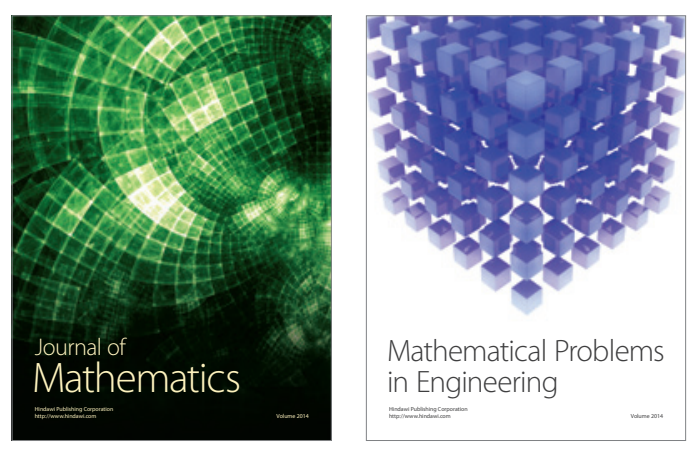

Mathematical Problems in Engineering
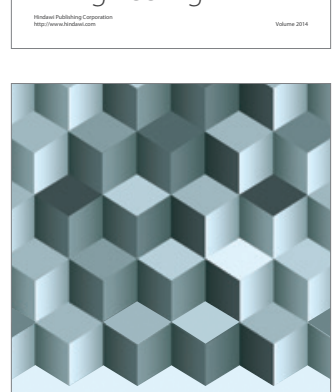

Journal of

Function Spaces
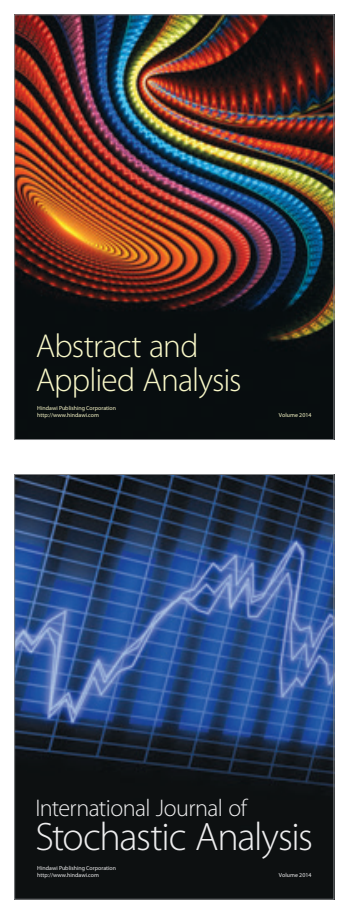

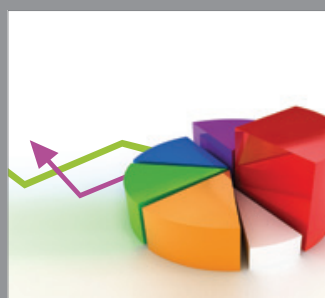

ournal of

Probability and Statistics

Promensencen
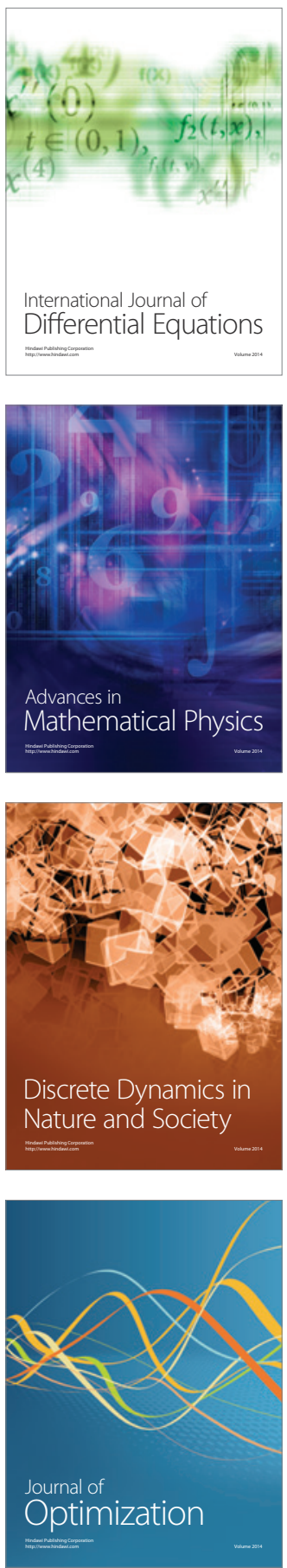